\title{
Oral-Facial Tissue Reconstruction in the Regenerative Axolotl
}

Andre Marc Charbonneau M.Sc ${ }^{1}$, Stephane Roy Ph.D2 ${ }^{*}$, Simon D Tran D.M.D., Ph.D ${ }^{1 *}$

${ }^{1}$ Craniofacial Tissue Engineering and Stem Cells Laboratory, Faculty of Dentistry, McGill University, Montreal, Canada, H3A 0C7.

${ }^{2}$ Department of Stomatology, University de Montreal, Montreal, Canada, H3C 3JT.

1 Table

6 Figures

2 Supplementary Figures

Short Running Title:

Axolotl Oral-Facial Tissue Regeneration

Key words: Wound Healing, Regeneration, Histology, Axolotl, Craniofacial Biology

*Correspondence to, S.Roy \& S.Tran. S.R @ U de M, 2900 Édouard Montpetit, Montréal, P.O Box 6128 Station Centre-Ville, H3C 3J7, Tel: 514-343-6111 ext 1780; Fax: 514-343-2237. Email: stephane.roy@umontreal.ca, S.T @ McGill University, Faculty of Dentistry, 3640 University Street, Montreal, H3A 0C7, Tel: 514-398-7203; Fax: 514-398-8900. Email: simon.tran@mcgill.ca 


\section{Abstract}

Absence of large amounts of orofacial tissues caused by cancerous resections, congenital defects or trauma result in sequelae such as dysphagia and noticeable scars. Oral-neck tissue regeneration was studied in the axolotl (regenerative amphibian) following a $2.5 \mathrm{~mm}$ punch biopsy that simultaneously removed skin, connective tissue, muscle, and cartilage in the tongue and intermandibular region. The untreated wound was studied macroscopically and histologically at 17 different time points ranging from $0-180 \mathrm{~d}(\mathrm{~N}=120$ axolotls $)$. At $12 \mathrm{~h}$ the wound's surface was smoothened and within 1mm, internal lingual muscular modifications occurred; at the same distance, between days 4-7 lingual muscle degradation was complete. Immunofluorescence indicates complete keratinocytes migration by $48 \mathrm{~h}$. These cells with epidermal Leydig cells, appearing yellow, lead the chin's deep tissue outgrowth until its closure on the $14^{\text {th }}$ day. Regeneration speeds varied and peaked in time for each tissue, 1) deep Immunofluorescence to Col IV showed basement membrane reconnected between days 30-45 coinciding with the chin's dermal tissue's surface area recovery. New muscle appeared at $21 \mathrm{~d}$ and was always preceded by the formation of a collagen bed. Both chin tissues regain all surface area and practically all components while the lingual structure lacks some content but is generally similar to the original. The methodology and high-resolution observations described here are the first of its kind for this animal model and could serve as a basis for future studies in oral and facial regenerative research. 


\section{Introduction}

\section{Objectives and Rationale}

Oral and neck tissue losses can be caused by surgical resection of cancers, congenital defects and trauma. Following reconstructive surgery, the reorganized tissue must continue to provide the patient with swallowing, breathing and communication abilities. This permanently visible region essential in our interactions with other people is one of the most challenging structures to reconstruct. Careful planning of the surgery and secondary intention healing is strongly encouraged in order to minimise side effects(Magill and Haughey, 2011). When secondary intention healing is not possible, microvascular free tissue transfers can often provide a functionally aesthetic replacement. This being said, lacunas are still present, namely: 1) an altered tissue texture; 2) scarring, weakened muscular ability and loss of sensation at the transplant and donor sites; 3) a patient's inadmissibility for surgery based on his clinical history; 4) tissue necrosis; and 5) infections(Navarro Cuellar et al., 2015). For these reasons, we believe it is important to investigate how tissue regeneration can be achieved in animal models in order to help solve oral-facial tissue reconstruction limitations in humans. A reproducible oral \& neck wound has been induced and investigated in the axolotl (Ambystoma mexicanum), an animal model known for its regenerative abilities(Roy and Levesque, 2006).

Regeneration in certain urodele (tailed) amphibians has been examined but their implementation has been restrained to the work of only several labs around the world(Roy and Gatien, 2008). To our knowledge, Goss and Stagg were the first to explore the regeneration in the head and neck regions of these animals(Goss and Stagg, 1958). They performed three different transverse amputation of a newt's lower jaw and after 6 months of observations, three different echelons of regeneration were observed. Amongst their observations, they noted degradation and 
restructuring of muscle near the wound margin and the formation of a blastema. The blastemal structure is a mass of cells found on most regenerating species and is the leading edge of the outgrowth. It is capped by the wound epithelium and is composed of what appears to be a homogenous cell types that can differentiate (Tanaka and Reddien, 2011). Soon after their original work, Goss and Stagg completed, a different type of amputation; they left the mandible bone intact but removed intermandibular soft tissues(Goss and Stagg, 1958). They described a thin closure of the wound followed by thickening. Unfortunately in those years, modern histological imaging could not be completed and to our knowledge, no other recent study has specifically explored histologically the regeneration of the intermandibular soft tissues in urodele amphibians. Ferretti's 1996 review discussed small advancements in the field and recapped some of the work produced by Goss and Stagg (Ferretti, 1996). Later, Ghosh used antibody labeling to investigate the origin of the cells inside the blastema of an amputated jaw(Ghosh et al., 1994). Nerves, striated muscles, cartilage, buccal epithelial cells were not present in the blastema but markers for dermal fibroblast and dental pulp were identified as blastemal contributors. Keratin positive cells were also observed in the wound epithelium providing evidence of their contribution in the process. Kurosaka's paper used 3D imaging to compare the healing after jaw amputation in regenerating newts and non-regenerating Xenopus. Differences between species were observed regarding BrdU incorporation and transcriptional and proteomic analysis of certain genes(Kurosaka et al., 2008).

Even though recent studies have focused specifically on oral wound healing(Larjava, 2012), none have explored the lingual and intermandibular structures simultaneous regeneration process. By resecting tissues in the axolotl's oral and neck areas using a punch biopsy, we hope to modernise the available data and provide new insights as to the main events occurring during regrowth of oral and neck tissues in this unique model organism. The comprehension of soft 
tissue regeneration is essential to a less invasive reconstruction reducing patient pain, hospital care time and un-esthetical outcomes.

\section{Methods}

\section{Animal Maintenance, Wounding and Tissue Processing:}

Overall $\sim 120$ wild type (majority) or white axolotls (Ambystoma mexicanum) (Ambystoma Genetic Stock Center Lexington, KY) were studied. They were bathed in 20\% v/v Holtfreter's solution (0.3mM $\quad \mathrm{MgSO}_{4} \quad 7 \mathrm{H}_{2} \mathrm{O}$ (Fischer-Scientific, BP213-1), $0.2 \mathrm{mM} \quad \mathrm{CaCl}_{2}$ (Fischer-Scientific, BP510), 0.17mM KCl (Fischer-Scientific, BP366), 12.5mM NaCl (FischerScientific, BP358), 0.45mM Trizma (Sigma Aldrich, T1753) (temp $=19-22^{\circ} \mathrm{C}, 12 \mathrm{~h}$ illumination). Pre-op animals were 8-10cm long. Anaesthesia involved submersion in MS-222 (Sigma Aldrich, E10S21) $7.7 \mathrm{mM}$ (diluted in Holtfreter's 20\% $\mathrm{pH} 7.0$ ) for 10min. An in-house constructed apparatus built from a $15 \mathrm{ml}$ plastic tube cut and thermally fused facilitated image capture pre and post-op. During the surgery, hydration of the gills and skin was intermittently performed. The punch biopsy $2.5 \mathrm{~mm}$ (Miltex) was inserted in approximately the identical location in each animal. The triangular target was based on anatomical pigmented blood vessels on the chin Postop, axolotls were re-immersed in fresh Holtfreter's, which over the next three days was changed daily. Euthanasia involved anaesthesia, decapitation (razor blade), isolation of the lower jaw tissue and fixation in paraformaldehyde $4 \%$. PBS rinses were completed prior to processing (Dehydrator Leica TP1020, paraffin embedding Shandom Histocentre 2 6400012). Sagittal sections at every $100 \mu \mathrm{m}$ from lateral mandible to medial line of the animal's lower jaw where mounted on glass slides and stained enabling visualisation of all sides of the wound and intact 
tissues surrounding it. The animal care and experiments were performed in accordance with the Université de Montréal animal care committee’s guidelines.

\section{Wound surface area and closure speed calculations:}

Each wounded animal was photographed on its chin and dorsal side of the tongue at 7.1X and 12.5X with the Leica MZ16F stereomicroscope. At each time point, 4-11 animals were selected for wound surface area measurements. Based on a ruler placed in certain images we created a digital scale bar enabling conversion of metric units to pixels for each magnification. Images were uploaded to Photoshop and pixel measurements were taken with the ruler tool. Surface area calculation of the superficial skin and deeper chin tissue was calculated based on an ellipse formula since it is the best representative shape of the wound in that location; for the tongue, calculations were based on a half-ellipse. To validate our measurements, surface areas in pixels of both magnifications were averaged and converted back to metric units to give one surface area value. The graph was computed using R softwares' EnvStats package with the stripChart code. The bars in the graph show confidence interval at $95 \%$ confidence with a nonparametrical analysis. The p-values between wounding locations at identical time points were computed using the R software and the wilcox.test code for a Wilcox Ranked Sum analysis. The speeds of the different tissues progressing to fill the voids found in Table 1 are calculated following this equation. ((Average Tissue Surface Area at Timepoint $\mathrm{X}+1)-($ Average Tissue Surface Area at Time X))/ time. The linear value is an approximation of a unidirectional closure speed based on a perfect circle and half perfect circle rather that ellipse and half ellipse. The pvalue evaluating the difference between surface areas in subsequent time points for the same wounded tissue seen in Figure 4 are demonstrated in Table 1 using the same statistical method as described above. 


\section{Sirius Red Chemical Stain:}

Tissue sections were rehydrated to water and soaked in Weigerts Hematoxlylin for 10min. The slides were then transferred to Sirius Red 0.1\% m/v (Direct Red 80 (Alfa Aesar, B21693) in a saturated solution of Picric Acid) for $1 \mathrm{~h}$. Two $5 \mathrm{~min}$ rinses of $0.5 \% \mathrm{v} / \mathrm{v}$ Acetic Acid ensued followed by rapid dehydration and addition of coverslip with organic mounting media. When taking the images in polarizing light (PL) the rotational angle of the stage was always adjusted to maximize the amount of teal coloration in the longitudinal muscle.

\section{Indirect Immunofluorescence staining:}

Rehydration of tissue to water followed by a heat induced epitope retrieval (20min at $\sim 95^{\circ} \mathrm{C}$ ) in $0.1 \mathrm{M}$ citrate at $\mathrm{pH}$ 6.0. A Universal blocking agent $1 \mathrm{X}$ (BioGenex, HK085-5K) was applied for $10 \mathrm{~min}$. Incubation of the antibodies was performed overnight at $4^{\circ} \mathrm{C}$ with both primary antibodies; these included, Collagen Type IV (AbCam, Ab 6586) 1:500 dilutions mixed at 1:1 with 100\% Anti-Pan Cytokeratin (AE1/AE3) pre-diluted solution (AbCam, Ab 961). The next morning, rinsing (all performed with PBS 1X) was succeeded by the addition of both secondary antibodies at 1:1 ratio with 1:400 Anti-Rabbit (Bio-Rad, 170-6515) and 1:100 AntiMouse (Life-Tech, A11029). The final results were found to be optimal with an incubation of the secondaries for $1 \mathrm{~h}-1 \mathrm{~h} 15 \mathrm{~min}$ (in the dark, room temp). Additional rinsing ensued followed by the addition of signal enhancer 1X Tyramide (Biotium, 92175), which incubated for 8-10min in dark. Another rinse was completed before the addition of DAPI followed by a final rinse before the addition of coverslips mounted with PBS 1X. The final mounting was with Prolong (Life Tech, P36931) but was only completed after the imaging since we felt it decrease the fluorescent intensity. Colour contrasts of the entire image were enhanced with Photoshop. 


\section{Imaging:}

Macroscopic images were taken at 7.1X and 12.5X with Leica MZ16F stereomicroscope. For microscopic images, an Axio-Imager M2 (Zeiss) microscope with a motorized stage captured normal and fluorescence images using 100X and 200X magnifications and were stitched by Zen Blue software (Carl Zeiss) to create a mosaic. Axiophot (Zeiss, 506747) was used with polarizing lenses and analyser slide (Zeiss, 433605-0000-000) to capture 25X images by manual tiling of the Sirius red stained slides; in this case stitching was performed with Photoshop.

\section{Results}

Chin wound closure after 14 days while tongue's regeneration is well advanced but not visually flawless at 180 days

Pictures were taken from several different angles for each time point to characterise the tissue regeneration process. Post-surgery, no severe haemorrhage was observed on the skin side or intra-orally The initial two dimensional surface area removed by the punch was calculated. For the skin, deep intermandibular tissue, and tongue the average surface areas were respectfully, $4240000 \mu \mathrm{m}^{2}, 3290000 \mu \mathrm{m}^{2}$ and $3300000 \mu \mathrm{m}^{2}$ Inside the cylindrical wound, unevenness from the cut muscle and skin layers were noticeable until smoothening of the wound occurred near $12 \mathrm{~h}$ (Fig $2 \mathrm{~T}=30 \mathrm{~min}-12 \mathrm{~h}$ Chin). A few hours after this time point, some animals showed a yellowish substance on the margins of the wound. This yellow substance composed mainly of keratinocytes and epidermal Leydig cells increased in intensity and formed a ring around the wound From $6 \mathrm{~h}$ to $7 \mathrm{~d}$, the blood vessels surrounding the wounds appeared redder and increased in intensity until a peak at $48 \mathrm{~h}$ and slowly decreased until the $7^{\text {th }}$ day Between $24-48 \mathrm{~h}$, yellow protrusions extended 
from the circumference over the wound hole This internal chin tissue in the hole of the chin concentrically closed after $14 \mathrm{~d}$ and was lead by the yellow mass of cells This internal tissue's highest closure speed was found between at 24 and $48 \mathrm{~h}$ but also extended to the 96h with an average closing speed of $23707 \mu \mathrm{m}^{2} / \mathrm{h}(84.3 \mu \mathrm{m} / \mathrm{h}$ unidirectional) over 3 days. Afterwards from 96h-14days the closing speed reached a stable progress averaging 7 $796 \mu \mathrm{m}^{2} / \mathrm{h}$ ( In this tissue, all adjacent compared time points including $24 \mathrm{~h}$ and onwards were significantly different in their surface area measurements (Table 1). Once the chin had closed, a shiny substance remained ventrally visible until the end of the time course. Blood vessels were seen entering the newly formed tissue The most external surface of the chin representing the skin layers had not yet closed; a slight unevenness between wounded and unwounded layers enabled visualising the differences and further quantification. Between days 0 and $7 \mathrm{~d}$ no significant progress was noted on this tissue (Table 1). Following this latency period, a peek speed was reached for 7 days between days 7-14 averaging 16 $139 \mu \mathrm{m}^{2} / \mathrm{h}(71.7 \mu \mathrm{m} / \mathrm{h}$ unidirectional). Slower average speeds were thereafter observed until complete closure between the $30^{\text {th }}$ and $45^{\text {th }}$ day (Fig. 2). The intraoral (tongue's) regeneration was quantified using a half ellipse model rather than a full ellipse like the two other tissues. Since this tissue was most often affected by slightly more than half the cylindrical punch biopsy tool we thought this would be more accurate. Its regenerated surface area did not show any statistical difference between time points $0-48 \mathrm{~h}, 7-14 \mathrm{~d}$ and 30-60days The earlier time points progress at a speed of $5670 \mu \mathrm{m}^{2} / \mathrm{h}$ and the later at 2 $649 \mu \mathrm{m}^{2} / \mathrm{h}$. Most importantly though, an abrupt increase of speed to $11604 \mu \mathrm{m}^{2} / \mathrm{h}(86 \mu \mathrm{m} / \mathrm{h}$ unidirectional) is noted for 5 days between time $48 \mathrm{~h}$ and $7 \mathrm{~d}$ but is followed by a total absence of progress from days 7-14 (After the 14th day, two dimensional significant surface 
area differences were only noted in this tissue until $\mathrm{T}=30 \mathrm{~d}$. On days $14-30$ a concentration of yellow substance was observable in the cleft of the lingual The tongue always grew outwards from the posterior and medial side of the wound. A thinner regrown replacement of the lingual structure attained a maximum near symmetry on the $45^{\text {th }}$ day and its thickening seemingly ensued At the $180^{\text {th }}$ day the re-establishment of most of the contour and thickness could be noticed interestingly, a cartilage gap inside the tongue remained Dermal pigmentation and vascularization of the regenerated tissue were observed. The extraoral wounded side on the chin sometime had a more shiny appearance than its control side.

Muscle regeneration started with a degradation phase until the $7^{\text {th }}$ day; onwards, the reconstruction phase unfolded and was supported by a collagen bed.

To further investigate the macroscopic observations, the Sirius Red histological chemical stain was used to assess collagen(Junqueira et al., 1978). With this staining method, two different types of lighting, bright light (BL) and polarized light (PL) could be used to evaluate the tissue content. Viewing of is strongly encouraged as it is a cartoon representation of the events.

Following the injury, a large gap was observed in the sagittal sections taken from the middle of the wound. In more medial or lateral sections, a blunt end of the hyoid apparatus was observed Muscles remained close to the wound margin but within the next 12 hours, histological modifications occurred. This was best observed in where the longitudinal muscle lost its teal colour to a distance of $\sim 1 \mathrm{~mm}$ from the wound margin. In BL, a difference in structure rather than color was consistently observed following $30 \mathrm{~min}$ At $12 \mathrm{~h}$ the cellular content visually seemed to increase continuously in the changing muscle and at $48 \mathrm{~h}$ 
both posterior and anterior wound surfaces were covered completely by a thin layer of cells. In BL, the yellowness in the muscle slowly faded and disappears between the $96 \mathrm{~h}$ and $7 \mathrm{~d}$ to the extent of the previously described teal color recession At 14 days it is noteworthy to see the first cells contacting to close the gap created by the punch Amongst the cell types, Leydig cells(Jarial, 1989, Farkas and Monaghan, 2015) (large amphibians epithelial cells) are identified by their large granular morphology and are seen filling the gap between the anterior and posterior side of the wound. Between days 7 and 180, yellow, green and red filaments are observable in $\mathrm{PL}$, where the lingual muscle used to be; In $\mathrm{BL}$, red or pink filaments recapitulated this observation. The first appearance of new muscle cells in teal or yellow (PL or BL respectively) was at this point sporadically noticeable in the suspected collagen matrix composed of the colourful filaments On the $21^{\text {st }}$ day, the medial posterior side of the tongue has begun its outgrowth At 30 days near the skin's surface a thick orange band (corresponding to the basement membrane (BM)) sectioned at surgery had reconnected on both sides of the wound; some of it seemingly interacting with red coloured fibres (BL) in the collagen matrix now becoming the muscle forming bed Even though it was unorganized, transverse muscle started to reappear in the void left by the wound. The longitudinal muscle emerged from the red bed (BL) but also seemed to sprout from the more posterior intact muscle. Initially the muscles formed in the bed were not properly oriented but seemingly modified their orientation as time progressed. Between the $60^{\text {th }}$ and $180^{\text {th }}$ days, anterior progression of the muscle was observed This being said, incomplete cartilage regeneration was observed for the animal's hyoid apparatus in the tongue 
Complete keratinocytes migration occurred over the wound within $48 \mathrm{~h}$ and dermal basement membrane completely reconnected $45 \mathrm{~d}$ post op.

At $48 \mathrm{~h}$ in BL the Sirius Red stain showed a thin layer of cells that had migrated vertically over the wound surface ; an Anti-Pan Cytokeratin identified those cells as being keratinocytes. The cytokeratin panel antibody had abundant signal in all oral mucosa and on skin epithelia. Immediately after wounding, both anterior and posterior sides did not possess any keratinocytes covering the wound margins The anterior side was always the first to be covered. In all animals ( $n=4-8$ per time point) keratinocyte migration was completed over all wound surfaces within $48 \mathrm{~h}$ These keratin positive cells first and mainly covered the wound; in addition similar to previous studies(Hadorn and Chen, Chiakulas, 1952) Leydig cells from the epithelium also covered the wound surface These two cell types were the first to contact between both joining edges of the closing wound at 14 days

At 30 days, Sirius Red in PL showed the reconnection of a thick orange band passing under the layers of skin An Anti-Collagen Type IV antibody confirmed its identity as the BM. Collagen of type IV stained in major part only the thick line underlying the skin side and a thinner line was noted for oral cavity. The BM did not follow the keratinocytes by vertically covering the wound but rather stayed faithful to their previous horizontal trajectory. This thick collagen type IV positive line awaited wound closure to occur before progressing and joining the two sectioned ends at the 30 day mark At this point, only a thin amount connected both sides but the full replenishment was noted in all animals at 45 days Interestingly, the inner content of Leydig cells sometimes stained positive to Anti Col IV and green and red mixed emissions (causing yellow) were also detected in cells of the circulatory system 


\section{Discussion}

In this study we were able to: 1 ) show the effectiveness and reproducibility of a wounding process using a punch biopsy. 2) Obtain multiple angle high-resolution macroscopic time-course images of the wound healing process 3) calculate and compare tissue growth and speeds at different times in different tissues, and 4) visualize microscopically key cellular events throughout the $180 \mathrm{~d}$ regeneration process including, keratinocyte and Leydig cell migration, muscular turn around events and basement membrane reconnection.

Ziermann recently described the head and neck muscular anatomy of the axolotl. According to her work, the axolotl does not have a tongue with intrinsic muscles(Ziermann and Diogo, 2013) as humans do. Its apparent lingual structure should rather be interpreted as a more posterior structure in the human throat. Comparatively speaking, Ziermann associated the two main muscles wounded in this study to the stylohyoid and the posterior digastric muscles in humans; in the axolotl, they are the brachiohyoidus (parallel to the sagittal section) and intermandibularis posterior (perpendicular to the sagittal section), respectively(Ziermann and Diogo, 2013). Only through metamorphosis does a more anterior secondary structure appears possibly better resembling what we think as the human tongue(Greven et al., 2013). Even though the structure wounded in this study do not entirely correspond to the human tongue, the results described here-in can be used to discuss wounds of the mouth and neck.

In a pilot study we attempted different punch biopsy sizes. At $1.5 \mathrm{~mm}$ the tissue did not easily extract with the punch and larger than $2.5 \mathrm{~mm}$ yielded increased mortality. When 
comparing to surrounding tissues, the hole represented a removal of $\sim 30-50 \%$ of its tongue and covered $1 / 4$ of the diameter of his lower jaw.

Migration of the keratinocytes over the wound, is an event well known to be essential to limb regeneration(Moriyasu et al., 2012) and is observed in oral wound healing(Larjava, 2012). We also observed this migration to occur from the dorsal and ventral sides of the wound rather than emerging from the wound surface. Later accumulation of these cells formed a yellow mass that lead to hole closure on the chin and the anterior expansion of the tongue in the mouth. Interestingly, once the mass encountered a physical counter-stress i.e 1) closure of hole 2) contour shape regain of tongue, the yellow mass disappeared possibly linking its usefulness to the re-establishment of only the basic structure. Yellow pigment cells also appear following the amputation in the oral siphon of C. intestinalis(Auger et al., 2010) and lower jaw of zebra fish(Wang et al., 2012). This phenomenon in three different regenerating species wounded near their relative oral cavity suggests importance on this matter and further investigation into these cells' origin, role, fate and presence in other regenerating locations would be of interest in future studies.

The fastest surface area recoveries were noted for the internal and external chin tissue. The axolotl's tongue is thicker and composed of greater amounts of tissue in comparison to the chin tissues; therefore, this could be a possible explanation for its longer and incomplete regeneration. It is also noteworthy to consider the wound shapes. The wound in the chin layers were circular contrasting the semi-circle shape found on the tongue. In a circular wound, multidirectional growth is permitted while in the tongue's circumstance, only unidirectional extrusion based growth occurred. It would have been 
interesting to have two similar circular shaped wounds in the chin and tongue to compare. Interestingly, two circular shaped wounds are found in the chin; these include the wounds in the deep and superficial tissues. We previously considered that a thicker tissue construct like the tongue takes more time to regenerate but in the chin, the thicker internal tissue is faster to regain its surface area. We noted no significant progress in the superficial tissues' surface area recovery until the $7^{\text {th }}$ day while the internal layers show significant progress with the first $24 \mathrm{~h}$. This delay observed in the thinner superficial skin layer may be caused by the presence of major skin components such as the basement membrane.

The first event observed and noticeable only in PL was the modification of the teal color inside the longitudinal muscle (brachiohyoidus); within 12 hours this stained muscle looses its color as it darkens to a certain distance from the wounds margin. With Sirius Red, the only type of documented tissue that possesses some type of teal appearance is collagen type II found in cartilage(Junqueira, Cossermelli and Brentani, 1978) which muscle does not have(Hutchison et al., 2007). Following the removal of a large muscle across a critical gap in non-regenerating species it will not regenerate or heal(Stocum, 2006). The exposure of the inner muscular content is said to be the first event in traumatic muscle lesions. A calcium dependent mechanism leads to the activation of the degradation stage. Neutrophils and macrophages are often involved in cleaning the injury up to a distance of about $1-3 \mathrm{~mm}$ from the wounds(Milner and Cameron, 2013). This coincides with our observation of the fading teal color (within 12h) and muscular degradation seen most importantly between the $96^{\text {th }} \mathrm{h}$ and 7 day. Subsequent to the $7^{\text {th }}$ day, cellular density appears to have gradually increase in the wound and was replaced by a collagen bed that seemed to provide a stimulatory environment for muscle regeneration. 
The absence of cartilage regeneration is an imperfection in an animal known to regenerate most of its tissues perfectly. Hutchison et al. showed that when a gap of critical sized dimension is made in the bone of an axolotls' forearm without amputation, the structure would not regenerate(Hutchison, Pilote and Roy, 2007). Our observations were in cartilage rather than in bone and we noticed only a small amount of remodelling by rounding off of the jagged surface. Just as in Hutchison's work, an amputation reproduces the structure but an inability is noticed when a simple gap is formed.

A shiny substance remained visible on the chin from after the wound closure until the $180^{\text {th }}$ day . Different animals at the time showed various amounts of shiny material and its presence seem inversely proportional to the progress of the transverse muscle in the histological sections. It suggests that as the collagen bed disappears and is replaced by the muscles the reflectivity subsides. Possibly because of the animal size, the regeneration observed in this study was not as perfect as in limb or tail regeneration where amputation is often conducted on animals less than half the size.

A thick orange filament observed in PL with the Sirius Red staining was suspected to be the BM. Collagen type IV is known to be singularly found in the BM. Positive signal in the suspected BM confirmed our histological observation and demonstrated the BM's return to normal state was within 45 days. The collagen IV signal was for the most part contained to the BM. In certain tissue sections the labeling extends to Leydig cells where we suspect either tyramide processing or autofluorescence.

The surgical approach and the imaging data including all time points has brought forth extended information on the regeneration process which will hopefully provide a baseline for future studies. We believe this animal's ability to regenerate oral and neck 
wounds should be further investigated seeing as it regains an appreciable aesthetic appearance following a large traumatic lesion.

\section{Acknowledgements}

We are very thankful to Dr. Antonio Nanci and his team for the lending of equipment during the tissue processing and imaging of the histological samples. Additionally, a warm thank you to Dr. Peter Chauvin for his review of a preliminary version of this manuscript, and to Drs Ola Maria and Brennan Mui for preliminary work on the axolotls' secretory glands. The funding agencies supporting our research efforts were the Canadian Institutes of Health Research (CIHR) to SR and ST, the Réseau de Recherche en Santé Buccodentaire et Osseuse (RSBO) to SR and ST, McGill University Faculty of Dentistry, Saputo Family, Mrs. Pierrette Wong, the Dairy Farms of Canada and the Network for Canadian Oral Health Research (NCOHR). AC was the recipient of a CIHR scholarship. The authors declare no conflict of interest. 


\section{References}

Auger H, Sasakura Y, Joly J-S, Jeffery WR. 2010. Regeneration of oral siphon pigment organs in the ascidian Ciona intestinalis. Developmental Biology 339:374-389.

Chiakulas JJ. 1952. The role of tissue specificity in the healing of epithelial wounds. Journal of Experimental Zoology 121:383-417.

Farkas JE, Monaghan JR. 2015. Housing and Maintenance of Ambystoma mexicanum, the Mexican Axolotl. New York, NY: Springer New York.

Ferretti P. 1996. Re-examining jaw regeneration in urodeles: what have we learnt? The International journal of developmental biology 40:807-811.

Ghosh S, Thorogood P, Ferretti P. 1994. Regenerative capability of upper and lower jaws in the newt. The International journal of developmental biology 38:479-490.

Goss RJ, Stagg MW. 1958. Regeneration in lower jaws of newts after excision of the intermandibular regions. Journal of Experimental Zoology 137:1-11.

Greven H, Richter H, Clemen G. 2013. Formation of the secondary tongue in Hynobius leechi and Ambystoma mexicanum (Amphibia: Urodela). VERTEBRATE ZOOLOGY 63:217-232. Hadorn E, Chen PS. Beobachtungen über die Wanderung der Leydigschen Drüsenzellen bei Regenerationsvorgängen in der Larvenhaut vonTriton alpestris.

Hutchison C, Pilote M, Roy S. 2007. The axolotl limb: A model for bone development, regeneration and fracture healing. Bone 40:45-56.

Jarial MS. 1989. Fine structure of the epidermal Leydig cells in the axolotl Ambystoma mexicanum in relation to their function. Journal of Anatomy 167:95-102.

Junqueira LC, Cossermelli W, Brentani R. 1978. Differential staining of collagens type I, II and III by Sirius Red and polarization microscopy. Archivum histologicum Japonicum = Nihon soshikigaku kiroku 41:267-274. 
Kurosaka H, Takano-Yamamoto T, Yamashiro T, Agata K. 2008. Comparison of molecular and cellular events during lower jaw regeneration of newt (Cynops pyrrhogaster) and West African clawed frog (Xenopus tropicalis). Developmental dynamics : an official publication of the American Association of Anatomists 237:354-365.

Larjava H. 2012. Oral wound healing cell biology and clinical managementeditor^editors. Chichester, West Sussex: John Wiley \& Sons.

Magill C, Haughey B. 2011. Principles and New Approaches in Surgical Reconstruction. In: Bernier J editor^editors. Head and Neck Cancer: Springer New York. p. 483-495. Milner D, Cameron J. 2013. Muscle Repair and Regeneration: Stem Cells, Scaffolds, and the Contributions of Skeletal Muscle to Amphibian Limb Regeneration. In: Heber-Katz E, Stocum DL editor ${ }^{\wedge}$ editors. New Perspectives in Regeneration: Springer Berlin Heidelberg. p. 133-159. Moriyasu M, Makanae A, Satoh A. 2012. Spatiotemporal regulation of keratin 5 and 17 in the axolotl limb. Developmental Dynamics 241:1616-1624.

Navarro Cuellar I, González Otero T, Ruiz Cruz JA, Navarro Vila C. 2015. Reconstruction of Soft Tissue Defects Using Microsurgical Flaps. Cham: Springer International Publishing.

Roy S, Gatien S. 2008. Regeneration in axolotls: a model to aim for! Experimental Gerontology 43:968-973.

Roy S, Levesque M. 2006. Limb regeneration in axolotl: is it superhealing?

TheScientificWorldJournal 6 Suppl 1:12-25.

Stocum DL. 2006. 9 - Regeneration of Musculoskeletal Tissues. In: Stocum DL editor^editors. Regenerative Biology and Medicine. Burlington: Academic Press. p. 221-249.

Tanaka E, Reddien PW. 2011. The cellular basis for animal regeneration. Developmental cell 21:172-185.

Wang X, He H, Tang W, Zhang XA, Hua X, Yan J. 2012. Two origins of blastemal progenitors define blastemal regeneration of zebrafish lower jaw. PloS one 7:e45380.

Ziermann JM, Diogo R. 2013. Cranial muscle development in the model organism ambystoma mexicanum: implications for tetrapod and vertebrate comparative and evolutionary morphology and notes on ontogeny and phylogeny. Anatomical record (Hoboken, NJ : 2007) 296:1031-1048. 
Table 1: Numerical data demonstrating the different speeds at which different tissue progress for the tissue regeneration. All units are in $\mu \mathrm{m}^{2}$ except for the linear row in $\mu \mathrm{m}$ which gives an approximation of the unidirectional growth speed. Statistical difference of the surface areas from Figure 4 for each respective wound comparing adjacent time points for a $p$-value $<0.05$ is denoted by *. 
Figure Legends 
